\title{
Dominant and Complementary Multi-Emotional Facial Expression Recognition Using C-Support Vector Classification
}

\author{
Christer Loob $^{1}$, Pejman Rasti ${ }^{1}$, Iiris Lüsi ${ }^{1}$, Julio C. S. Jacques Junior ${ }^{2}$, Xavier Baró ${ }^{3}$, Sergio \\ Escalera $^{2}$, Tomasz Sapiński ${ }^{4}$, Dorota Kaminska ${ }^{4}$ and Gholamreza Anbarjafari ${ }^{1,5}$ \\ ${ }^{1}$ iCV Research Group, Institute of Technology, University of Tartu, Tartu 50411, Estonia \\ ${ }^{2}$ Human Pose Recovery and Behavior Analysis, University of Barcelona, Barcelona, Spain \\ ${ }^{3}$ Computer Vision Center and Universitat Oberta de Catalunya, Barcelona, Spain. \\ ${ }^{4}$ Institute of Mechatronics and Information Systems, \\ Lodz University of Technology, Lodz, Poland \\ ${ }^{5}$ Department of Electrical and Electronic Engineering, Hasan Kalyoncu University, Gaziantep, Turkey
}

\begin{abstract}
We are proposing a new facial expression recognition model which introduces 30+ detailed facial expressions recognisable by any artificial intelligence interacting with a human. Throughout this research, we introduce two categories for the emotions, namely, dominant emotions and complementary emotions. In this research paper the complementary emotion is recognised by using the eye region if the dominant emotion is angry, fearful or sad, and if the dominant emotion is disgust or happiness the complementary emotion is mainly conveyed by the mouth. In order to verify the tagged dominant and complementary emotions, randomly chosen people voted for the recognised multi-emotional facial expressions. The average results of voting are showing that $\mathbf{7 3 . 8 8 \%}$ of the voters agree on the correctness of the recognised multi-emotional facial expressions.
\end{abstract}

\section{INTRODUCTION}

Facial expressions are one of the most effective ways for humans to communicate due to the fact that they contain critical and necessary information regarding human affective states [1]-[4]. Automatic recognition of facial expressions is a complex issue because of significant variations in the physiognomy of faces with respect to person's identity, environment illumination and head pose [5], [6].

Automatic facial expression recognition is widely used in many applications such as assistive robotics, human emotion analysis, and human-machine interfaces [7], [8]. As a result, recognition of facial expressions has become an important research topic in human-computer interaction, as well as in the fields of machine learning, image processing, human cognition, and pattern recognition.

The general approach to facial expression recognition consists of 5 stages [9]: preprocessing, face acquisition, image segmentation, feature extraction and classification. Preprocessing consists of image enhancement techniques such noise removal or illumination enhancement [10]. The face acquisition involves utilisation of some face detection algorithm, such as the Viola-Jones [11] algorithm. The facial

This work is supported Estonian Research Council Grant (PUT638), the Estonian Centre of Excellence in IT (EXCITE) funded by the European Regional Development Fund, the Spanish Projects TIN2013-43478-P and TIN2016-74946-P (MINECO/FEDER, UE), CERCA Programme / Generalitat de Catalunya and the European Network on Integrating Vision and Language (iV\&L Net) ICT COST Action IC1307. components of regions of interest such as mouth, eyes, ears, cheeks, nose, forehead, and eyebrow are detected during the third step. At the fourth stage a feature extraction algorithm such as local binary patterns (LBP) [12], Gabor filters [13], linear discriminant analysis (LDA) [14], principal component analysis (PCA) [15], local gradient code (LGC) [16], and independent component analysis (ICA) [17] is used in order to extract the feature points from the regions of interest. Finally, a classification method is used to find the recognition rate. Some of the most popular classification methods are support vector machines (SVM) [18] and artificial neural networks (ANN) [19].

An automated Facial Action Coding System (FACS) system is presented by Jihun Hamm et al. [20]. In their work, dynamic changes of facial actions in neuropsychiatric patients' videos were analyzed by measuring the movements of facial action units. As mentioned by the authors, they approach has some advanges in comparison with other methods. It analyses dynamical expression changes through videos (instead of still images), it measures individual (and combined) facial muscle movements through Action Units (AUs), instead of a few prototypical expressions, and finally, it performs automatically without requiring interventions from an operator. In summary, geometric and texture features are automatically extracted from video and tracked, and then temporal profiles of facial muscle movements are produced.

Luo et al. [21] presented a facial expression recognition algorithm by combining PCA and LBP features in a SVM classifier. In their work, local features are extracted from the mouth region, which gives support the global features.

In this work, we present a new facial expression recognition model that uses illumination enhancement [22] and Voila-Jones face detection algorithms, as well as automatic facial feature extraction techniques and SVM for feature extraction and classification.

The remainder of this paper is organised as follows. A detailed overview of the proposed method is presented in Section II. Section III contains the outcome of experimental results. Finally, Section IV concludes the paper. 


\section{The Proposed Method}

Although most research works are focused on classical emotions which are anger, contempt, disgust, fear, happiness, sadness, and surprise as well as an eighth emotion referred to as neutral [23]-[30], in this work we are proposing a step forward and introducing further facial expression recognition, by dividing the facial expressions into two main categories, namely, dominant expressions and complementary expressions. Dominant expressions include the seven main expressions. The complementary expression are those minor expressions that people express while they are in one of the seven classical states. Based on the aforementioned basic emotions, one can have 30+ different expressions which are combinations of the seven basic expressions. These new expressions are referred to as surprisingly angry, disgustingly sad, happily surprised, and so on. The list of the possible emotions are given in Table I.

TABLE I

DOMINANT - COMPLEMENTARY EMOTION COMBINATIONS

\begin{tabular}{|l|c|c|c|c|c|c|c|}
\hline & Angry & Contempt & Disgust & Fear & Happy & Sadness & Surprise \\
\hline Angry & angry & $\begin{array}{c}\text { contemptly } \\
\text { angry }\end{array}$ & $\begin{array}{c}\text { disgustingly } \\
\text { angry }\end{array}$ & $\begin{array}{c}\text { fearfully } \\
\text { angry }\end{array}$ & $\begin{array}{c}\text { happily } \\
\text { angry }\end{array}$ & $\begin{array}{c}\text { sadly } \\
\text { angry }\end{array}$ & $\begin{array}{c}\text { surprisingly } \\
\text { angry }\end{array}$ \\
\hline Contempt & $\begin{array}{c}\text { angrily } \\
\text { contempt }\end{array}$ & contempt & $\begin{array}{c}\text { disgustingly } \\
\text { contempt }\end{array}$ & $\begin{array}{c}\text { fearfully } \\
\text { contempt }\end{array}$ & $\begin{array}{c}\text { happily } \\
\text { contempt }\end{array}$ & $\begin{array}{c}\text { sadly } \\
\text { contempt }\end{array}$ & $\begin{array}{c}\text { surprisingly } \\
\text { contempt }\end{array}$ \\
\hline Disgust & $\begin{array}{c}\text { angrily } \\
\text { disgusted }\end{array}$ & $\begin{array}{c}\text { contemptly } \\
\text { disgusted }\end{array}$ & disgust & $\begin{array}{c}\text { fearfully } \\
\text { disgusted }\end{array}$ & $\begin{array}{c}\text { happily } \\
\text { disgusted }\end{array}$ & $\begin{array}{c}\text { sadly } \\
\text { disgusted }\end{array}$ & $\begin{array}{c}\text { surprisingly } \\
\text { disgusted }\end{array}$ \\
\hline Fear & $\begin{array}{c}\text { angrily } \\
\text { fearful }\end{array}$ & $\begin{array}{c}\text { contemptly } \\
\text { fearful }\end{array}$ & $\begin{array}{c}\text { disgustingly } \\
\text { fearful }\end{array}$ & fearful & $\begin{array}{c}\text { happily } \\
\text { fearful }\end{array}$ & $\begin{array}{c}\text { sadly } \\
\text { fearful }\end{array}$ & $\begin{array}{c}\text { surprisingly } \\
\text { fearful }\end{array}$ \\
\hline Happy & $\begin{array}{c}\text { angrily } \\
\text { happy }\end{array}$ & $\begin{array}{c}\text { contemptly } \\
\text { happy }\end{array}$ & $\begin{array}{c}\text { disgustingly } \\
\text { happy }\end{array}$ & $\begin{array}{c}\text { fearfully } \\
\text { happy }\end{array}$ & happy & $\begin{array}{c}\text { sadly } \\
\text { happy }\end{array}$ & $\begin{array}{c}\text { surprisingly } \\
\text { happy }\end{array}$ \\
\hline Sadness & $\begin{array}{c}\text { angrily } \\
\text { sad }\end{array}$ & $\begin{array}{c}\text { contemptly } \\
\text { sad }\end{array}$ & $\begin{array}{c}\text { disgustingly } \\
\text { sad }\end{array}$ & $\begin{array}{c}\text { fearfully } \\
\text { sad }\end{array}$ & $\begin{array}{c}\text { happily } \\
\text { sad }\end{array}$ & sad & $\begin{array}{c}\text { surprisingly } \\
\text { sad }\end{array}$ \\
\hline Surprise & $\begin{array}{c}\text { angrily } \\
\text { surprised }\end{array}$ & $\begin{array}{c}\text { contemptly } \\
\text { surprised }\end{array}$ & $\begin{array}{c}\text { disgustingly } \\
\text { surprised }\end{array}$ & $\begin{array}{c}\text { fearfully } \\
\text { surprised }\end{array}$ & $\begin{array}{c}\text { happily } \\
\text { surprised }\end{array}$ & $\begin{array}{c}\text { sadly } \\
\text { surprised }\end{array}$ & surprised \\
\hline
\end{tabular}

By introducing such detailed facial expression recognition technique, a human-computer interaction system will be able to understand the emotional state of the user more accurately and make a more appropriate decision. The dominant facial expression is recognised by using all features and detected landmarks on facial images, whereas the complementary expressions have been recognised only by using lower facial landmarks.

The general steps of the proposed facial multi-emotional recognition system is shown in Fig. 1. The details of each part is explained below as well.

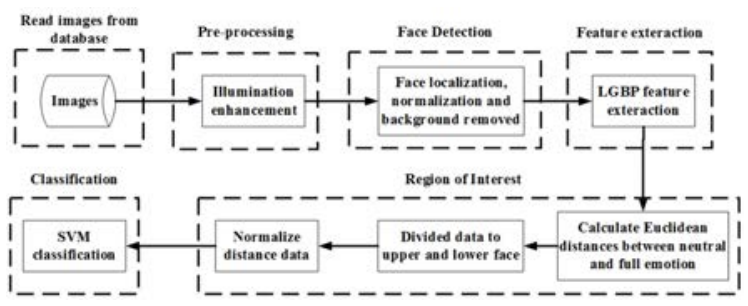

Fig. 1. Flowchart of the proposed method.

\section{A. Feature extraction}

In the proposed method we extract 68 intransigent feature points including 6 points for both eyes, 5 points for both eyebrows, 9 points for the nose, 20 points for the mouth region. The remaining 17 extracted points represent the boundaries of the face. For feature extraction, we adopted the FERA 2015 baseline system described in [31] and code by Baltrusaitis [32]. The face is segmented so that the eyes, eyebrows, nose, 6 of the upper boundary points are considered as upper face points and rest as lower face points.

It should be mentioned that number of extracted feature points are varied in different methods according to their purpose or their limitation such as 34 points in [33] or 24 facial points in [34].

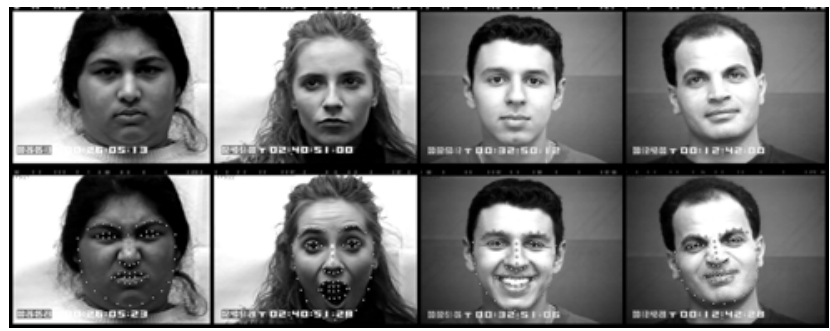

Fig. 2. Extracted feature points.

Fig. 2 shows some sample facial images with their extracted features marked. In the proposed method faces on the images in the dataset are first detected using Viola \& Jones face detector [35]. The images are then normalized in terms of size, translation, rotation and the background is removed. Only prelabelled image sets are used and all images excluding neutral and full emotion samples are removed from further processing.

The feature extraction process begins by grayscaling the images. Face localisation and segmentation is accomplished using the Viola \& Jones face detector [35]. The image is split into a $4 \times 4$ grid to maintain some local information. Appearance features are extracted using Local Gabor Binary Patterns (LGBP) due to its robustness to complex variations in expression and illumination [36]. The image is first convolved with a set of Gabor filters after which LBP feature extraction occurs over the set of Gabor magnitude response images [31], [36].

Euclidean distances between corresponding points on neutral and full emotion images are calculated. The resulting data is normalised and sent to the support vector machine for dominant emotion classification. The data is then divided into two data sets describing the lower- and the upper half of the face. The resulting distances are normalised and distances below 0.2 will be eliminated. The data is then sent to a support vector machine for complementary emotion classification.

\section{B. C-support vector classification}

We approach the problem by using the leave-one-out method by training on all the data except for the current test sample. The sample is classified by SVM a maximum of 7 times for the 7 basic emotions or until a successful 
classification of any emotion is obtained.

For this type of SVM training involves the minimisation of the error function:

$$
\frac{1}{2} w^{T} w+C \sum_{i=1}^{l} \xi_{i}
$$

Subject to the constraints:

$$
\begin{gathered}
y_{i}\left(w^{T} \phi\left(x_{i}\right)+b\right) \geq 1-\xi_{i}, \\
\xi_{i} \geq 0, i=1, \ldots, l,
\end{gathered}
$$

Where $w$ is the width of the hyperplane margin, $\mathrm{C}$ is the penalty parameter, the index i labels the 1 training cases, $\xi$ represents the independent variables and $y \in \pm 1$ represents the class labels. The kernel function $\phi$ is used to transfrom $\operatorname{data}\left(x_{i}\right)$ from the input to the feature space. The kernel function [37] is also called $K\left(x_{i}, x_{j}\right) \equiv \phi\left(x_{i}\right)^{T} \phi\left(x_{j}\right)$.

The kernel function used for the proposed method is Radial Basis Function (RBF) kernel in which case:

$$
K\left(x_{i}, x_{j}\right)=\exp \left(-\gamma\left\|x_{i}-x_{j}\right\|^{2}\right), \gamma>0
$$

Where $\gamma$ represents the influence a single training sample has and $\left\|x_{i}-x_{j}\right\|^{2}$ is the squared Euclidean distance between the two feature vectors.

The kernel nonlinearly maps samples into a higher dimensional space so it can handle the case when the relation between class labels and attributes is nonlinear. Furthermore, the RBF kernel uses less hyperparameters, which influence the complexity of the model selection, than for example a polynomial kernel would have. The RBF kernel also has fewer numerical difficulties in contrast to polynomial kernels.

\footnotetext{
Algorithm 1 PROPOSED METHOD SCHEMA

Input: Facial images from database

Output: Emotional Recognition
}

Illumination enhancement for each image of database. localization of enhanced images.

Normalization of localized images.

Face detection by using Viola-Jones and remove background.

feature extraction by using LGBP.

Find the euclidean distance between neutral and full emotion.

Divided data to upper and lower face.

Find the recognition rate by using SVM.

\section{Dominant and complimentary expression}

It is clear that different regions of the face convey different types of affective information and some parts of the face convey some emotions better than the others. While there is some evidence that the eye region is more informative for human subjects in recognising angry, fearful and sad faces, disgust and happiness appears to be mainly conveyed by the mouth. Surprise may be similarly recognised from both regions. Human subjects recognise happiness from the bottom half of the face as accurately and even faster than from the whole face [38], [39].

Affective information is not distributed equally across the face and different emotions utilise different parts of the face. Blais [40] found that the mouth area appears to be the most informative part of the face when recognising emotions. The mouth region is the biggest region of the face in area and has the ability to move the most. Hence it is the most informational part of the human face [41].

Due to the intricacies of the human face the seven basic emotions are not nearly enough to cover the whole emotional range of a person. Emotions can often come in combinations, for example, a person can be both happy and surprised at the same time.

In an attempt to alleviate this problem we have utilised a complementary emotion in addition to the dominant one. In the proposed method, the complementary emotion is extracted from the either the lower or upper half of the face while the information about the dominant emotion is extracted from the whole face. If the dominant emotion is classified as one of the emotions that is mainly influenced by the upper part of the face (anger, fear, sadness), then we search for the complementary emotion in the lower half of the face and vice versa.

Such method allows to classify an expression as a combination of two emotions and provides us with $30+$ variations, if we classify for seven basic emotions. If the dominant emotion is classified as 'happy' and the complementary emotion as 'surprise', we would call the resulting emotion combination 'Surprisingly happy'. The experimental results, reported in the Section III, show that not all 49 multiemotional expression exist. However the results state that the proposed method can always recognises $30+$ emotions.

\section{The EXPERIMENTAl Results AND Discussions}

In this work Cohn-Kanade AU-Coded Facial Expression $(\mathrm{CK}+)$ database [42], [43] and Japanese Female Facial Expression (JAFFE) image database [44], [45] have been used in order to conduct the experimental results. The first database contains 327 samples of the 8 facial expressions (seven basic facial expressions an a neutral expression) of 100 distinctive people. The second database contains 213 images of 7 facial expressions (six basic facial expressions and one neutral) posed by 10 Japanese female models.

The results of applying the proposed method on all of the faces in $\mathrm{CK}+$ database are shown in Table II. It has the recognition rate of $100 \%$ for angry, happy and surprised emotion. Minimum recognition rates belong to contempt and sadness emotions.

Table III illustrates the recognition rates of complimentary emotions for all available dominant emotions in the $\mathrm{CK}+$ database. The results are a combination of what proposed in [40] and [39]. Results marked in bold are considered to be 
TABLE II

THE DOMINANT EMOTION FOR THE CK+ DATABASE

\begin{tabular}{|l|c|}
\hline & $\begin{array}{c}\text { Recognition } \\
\text { Rate }(\%)\end{array}$ \\
\hline Angry & 100 \\
\hline Contempt & 89 \\
\hline Disgust & 98 \\
\hline Fear & 96 \\
\hline Happy & 100 \\
\hline Sadness & 89 \\
\hline Surprise & 100 \\
\hline
\end{tabular}

pure emotions as the dominant and complementary emotions overlap.

\section{TABLE III}

ANGRY, FEAR, SADNESS GET COMPLEMENTARY EMOTION FROM LOWER FACE AND CONTEMPT, DISGUST, HAPPY, SURPRISE FROM UPPER FACE FOR THE CK+ DATABASE.

\begin{tabular}{|l|c|c|c|c|c|c|c|}
\hline & Angry & Contempt & Disgust & Fear & Happy & Sadness & Surprise \\
\hline Angry & $\mathbf{3 4}$ & 3 & 1 & 2 & 1 & 2 & 2 \\
\hline Contempt & 3 & $\mathbf{8}$ & 1 & 1 & 0 & 3 & 2 \\
\hline Disgust & 7 & 2 & $\mathbf{4 9}$ & 1 & 0 & 0 & 0 \\
\hline Fear & 3 & 1 & 1 & $\mathbf{1 4}$ & 2 & 1 & 3 \\
\hline Happy & 3 & 2 & 4 & 5 & $\mathbf{5 1}$ & 2 & 2 \\
\hline Sadness & 4 & 1 & 2 & 3 & 0 & $\mathbf{1 5}$ & 3 \\
\hline Surprise & 5 & 2 & 3 & 3 & 2 & 3 & $\mathbf{6 5}$ \\
\hline
\end{tabular}

The emotion recognition rates that is shown in Table IV and Table $\mathrm{V}$ are less than those for $\mathrm{CK}+$ database. In this database, most recognised images belong to the happy emotion category.

In order to have quantitative results on the proposed dominant and complementary emotion recognition, sets of images with the recognised labels, as shown in Figs 3, 4, 5 and 6, from $\mathrm{CK}+$ and JAFFE databases were shown to randomly chosen 64 people. They were asked to vote whether the recognised dominant and complementary emotion have been recognised correctly or not. The voting results showed that $73.88 \%$ of the people agreed on the tagged dominant and complementary emotions by the proposed method. The results of the voting is shown in Fig. 7. As it is illustrated in Fig. 7, except for angrily fearful in both CK+ and JAFFE databases the majority of the voters agreed on the correct tagging by the proposed method.

TABLE IV

THE DOMINANT EMOTION FOR THE JAFFE DATABASE.

\begin{tabular}{|l|c|}
\hline & $\begin{array}{c}\text { Recognition } \\
\text { Rate }(\%)\end{array}$ \\
\hline Angry & 67 \\
\hline Disgust & 55 \\
\hline Fear & 84 \\
\hline Happy & 87 \\
\hline Sadness & 45 \\
\hline Surprise & 73 \\
\hline
\end{tabular}

\section{CONCLUSION}

In this paper, a new methodology was developed for recognising more than the seven classical emotions by introducing
TABLE V

ANGRY, FEAR, SADNESS GET COMPLEMENTARY EMOTION FROM LOWER FACE AND DISGUST, HAPPY, SURPRISE FROM UPPER FACE FOR THE JAFFE DATABASE.

\begin{tabular}{|l|c|c|c|c|c|c|}
\hline & Angry & Disgust & Fear & Happy & Sadness & Surprise \\
\hline Angry & $\mathbf{1 9}$ & 3 & 1 & 0 & 2 & 1 \\
\hline Disgust & 0 & $\mathbf{1 8}$ & 3 & 0 & 1 & 0 \\
\hline Fear & 2 & 3 & $\mathbf{1 5}$ & 1 & 1 & 1 \\
\hline Happy & 0 & 0 & 1 & $\mathbf{2 5}$ & 2 & 2 \\
\hline Sadness & 4 & 0 & 0 & 1 & $\mathbf{1 6}$ & 2 \\
\hline Surprise & 1 & 0 & 0 & 2 & 2 & $\mathbf{2 0}$ \\
\hline
\end{tabular}

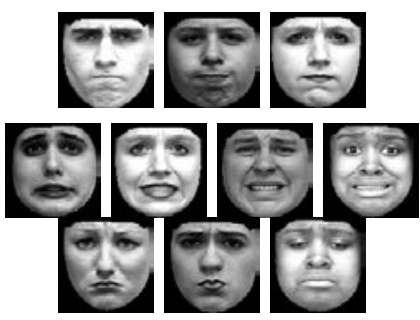

Fig. 3. Emotion pairs with the dominant emotions being angry, fear and sadness respectively for each row, complementary emotions are recognised from the lower part of the face for the $\mathrm{CK}+$ database. The first row illustrates from left to right emotion pairs angry, fearfully angry and surprisingly angry. The second row illustrates from left to right emotion pairs angrily feared, fear, happily feared and sadly feared. The third row illustrates from left to right emotion pairs angrily sad, fearfully sad and sad. Due to copyright issues all example emotion pairs from $\mathrm{CK}+$ database are not displayable.

the concept of a complementary emotion. In this research, the focus was on automatic recognition of a complementary emotion in addition to the dominant emotion. This research has accomplished recognising complementary emotions from samples labelled for only dominant emotions illustrating that the emotional range of a person is much greater than seven basic emotions. The developed methodology has been tested on existing datasets but to our knowledge there is currently no database with labels for complementary emotions to compare the results with.

We hope to pave way for new research by the introduction of the complementary emotion concept. Creation of a database with labels for both dominant and complementary emotions would greatly promote research in this area. Inclusion of psychologists in future research would provide deeper insight into human emotions.

\section{REFERENCES}

[1] R. Adolphs, "Cognitive neuroscience of human social behaviour," Nature Reviews Neuroscience, vol. 4, no. 3, pp. 165-178, 2003.

[2] L. Zhang and D. Tjondronegoro, "Facial expression recognition using facial movement features," Affective Computing, IEEE Transactions on, vol. 2, no. 4, pp. 219-229, 2011.

[3] F. Mormann, J. Niediek, O. Tudusciuc, C. M. Quesada, V. A. Coenen, C. E. Elger, and R. Adolphs, "Neurons in the human amygdala encode face identity, but not gaze direction," Nature neuroscience, 2015.

[4] C. Nagaraju, D. Sharadamani, C. Maheswari, and D. Vishnu Vardhan, "Evaluation of lbp-based facial emotions recognition techniques to make consistent decisions," International Journal of Pattern Recognition and Artificial Intelligence, vol. 29, no. 06, p. 1556008, 2015.

[5] W. Gu, C. Xiang, Y. Venkatesh, D. Huang, and H. Lin, "Facial expression recognition using radial encoding of local gabor features and classifier synthesis," Pattern Recognition, vol. 45, no. 1, pp. 8091, 2012. 


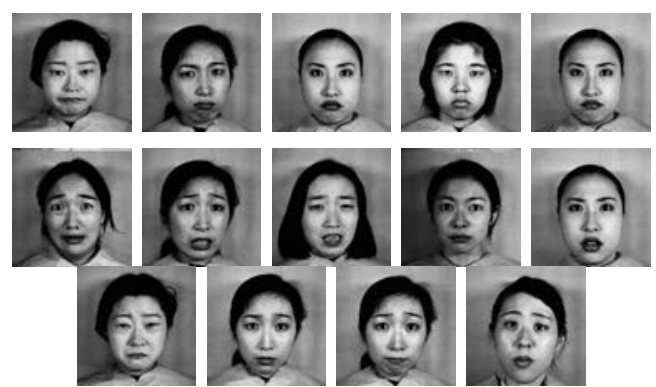

Fig. 4. Emotion pairs with the dominant emotions being angry, fear and sadness respectively for each row, complementary emotions are recognised from the lower part of the face for the JAFFE database. The first row illustrates from left to right emotion pairs angry, disgustingly angry, fearfully angry, sadly angry and surprisingly angry. The second row from left to right illustrates emotion pairs fearfully angry, disgustingly feared, fear, sadly feared and surprisingly feared. The third row illustrates from left to right emotion pairs angrily sad, happily sad, sad and surprisingly sad.

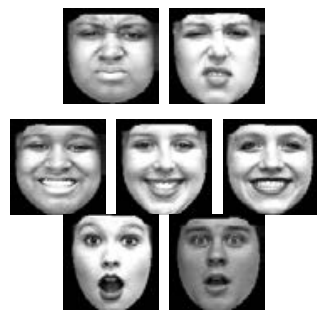

Fig. 5. Emotion pairs with the dominant emotions being disgust, happy and surprise respectively for each row, complementary emotions are recognised from the upper part of the face for the CK+ database. The first row illustrates from left to right emotion pairs angrily disgusted and disgust. The second row illustrates from left to right emotion pairs fearfully happy, happy and surprising happy. The third row illustrates from left to right emotion pairs fearfully surprised and sadly surprised. Due to copyright issues all example emotion pairs from $\mathrm{CK}+$ are not displayable.

[6] Y. Rahulamathavan, R. C.-W. Phan, J. Chambers, D. J. Parish et al., "Facial expression recognition in the encrypted domain based on local fisher discriminant analysis," Affective Computing, IEEE Transactions on, vol. 4, no. 1, pp. 83-92, 2013.

[7] Y. Dai, Y. Shibata, T. Ishii, K. Hashimoto, K. Katamachi, K. Noguchi, N. Kakizaki, and D. Cai, "An associate memory model of facial expressions and its application in facial expression recognition of patients on bed," in null. IEEE, 2001, p. 151.

[8] G. Anbarjafari and A. Aabloo, "Expression recognition by using facial and vocal expressions," $V \& L$ Net 2014, p. 103, 2014.

[9] J. Kumari, R. Rajesh, and K. Pooja, "Facial expression recognition: A survey," Procedia Computer Science, vol. 58, pp. 486-491, 2015.

[10] G. Anbarjafari, "Face recognition using color local binary pattern from mutually independent color channels," EURASIP Journal on Image and Video Processing, vol. 2013, no. 1, pp. 1-11, 2013

[11] P. Viola and M. J. Jones, "Robust real-time face detection," International journal of computer vision, vol. 57, no. 2, pp. 137-154, 2004.

[12] T. Ojala, M. Pietikäinen, and D. Harwood, "A comparative study of texture measures with classification based on featured distributions," Pattern recognition, vol. 29, no. 1, pp. 51-59, 1996.

[13] M. J. Lyons, J. Budynek, and S. Akamatsu, "Automatic classification of single facial images," IEEE Transactions on Pattern Analysis \& Machine Intelligence, no. 12, pp. 1357-1362, 1999.

[14] P. N. Belhumeur, J. P. Hespanha, and D. J. Kriegman, "Eigenfaces vs. fisherfaces: Recognition using class specific linear projection," Pattern Analysis and Machine Intelligence, IEEE Transactions on, vol. 19, no. 7, pp. 711-720, 1997.

[15] M. Turk, A. P. Pentland et al., "Face recognition using eigenfaces," in Computer Vision and Pattern Recognition, 1991. Proceedings

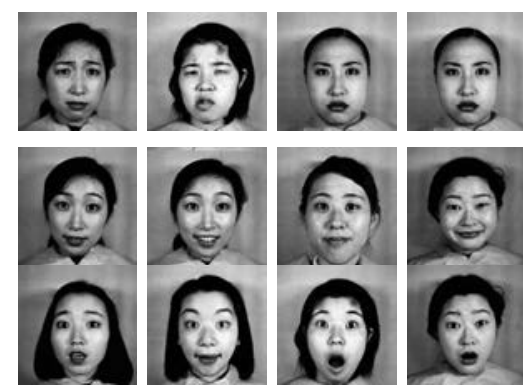

Fig. 6. Emotion pairs with the dominant emotions being disgust, happy and surprise respectively for each row, complementary emotions are recognised from the upper part of the face for the JAFFE database. The first row illustrates from left to right emotion pairs disgust, fearfully disgusted, sadly disgusted and surprisingly disgusted. The second row illustrates from left to right emotion pairs fearfully happy, happy, sadly happy and surprisingly happy. The third row illustrates from left to right emotion pairs angrily surprised, happily surprised, sadly surprised and surprise.

CVPR'91., IEEE Computer Society Conference on. IEEE, 1991, pp. $586-591$.

[16] Y. Tong, R. Chen, and Y. Cheng, "Facial expression recognition algorithm using lgc based on horizontal and diagonal prior principle," Optik-International Journal for Light and Electron Optics, vol. 125, no. 16, pp. 4186-4189, 2014

[17] M. S. Bartlett, J. R. Movellan, and T. J. Sejnowski, "Face recognition by independent component analysis," Neural Networks, IEEE Transactions on, vol. 13, no. 6, pp. 1450-1464, 2002.

[18] C.-W. Hsu, C.-C. Chang, C.-J. Lin et al., "A practical guide to support vector classification," 2003

[19] N. S. Altman, "An introduction to kernel and nearest-neighbor nonparametric regression," The American Statistician, vol. 46, no. 3, pp. 175-185, 1992.

[20] J. Hamm, C. G. Kohler, R. C. Gur, and R. Verma, "Automated facial action coding system for dynamic analysis of facial expressions in neuropsychiatric disorders," Journal of neuroscience methods, vol. 200, no. 2, pp. 237-256, 2011.

[21] Y. Luo, C.-m. Wu, and Y. Zhang, "Facial expression recognition based on fusion feature of pca and lbp with svm," Optik-International Journal for Light and Electron Optics, vol. 124, no. 17, pp. 27672770, 2013.

[22] G. Anbarjafari, A. Jafari, M. N. S. Jahromi, C. Ozcinar, and H. Demirel, "Image illumination enhancement with an objective noreference measure of illumination assessment based on gaussian distribution mapping," Engineering Science and Technology, an International Journal, vol. 18, no. 4, pp. 696 - 703, 2015. [Online]. Available: http://www.sciencedirect.com/science/article/pii/S2215098615000713

[23] P. Ekman and W. V. Friesen, Unmasking the face: A guide to recognizing emotions from facial clues. Ishk, 2003.

[24] F. Y. Shih, C.-F. Chuang, and P. S. Wang, "Performance comparisons of facial expression recognition in jaffe database," International Journal of Pattern Recognition and Artificial Intelligence, vol. 22, no. 03, pp. 445-459, 2008

[25] J. Liang, C. Xu, Z. Feng, and X. Ma, "Hidden markov model decision forest for dynamic facial expression recognition," International Journal of Pattern Recognition and Artificial Intelligence, vol. 29, no. 07, p. $1556010,2015$.

[26] P. Ekman, "Darwin, deception, and facial expression," Annals of the New York Academy of Sciences, vol. 1000, no. 1, pp. 205-221, 2003.

[27] H. Rosenberg, S. McDonald, M. Dethier, R. P. Kessels, and R. F. Westbrook, "Facial emotion recognition deficits following moderatesevere traumatic brain injury (tbi): Re-examining the valence effect and the role of emotion intensity," Journal of the International Neuropsychological Society, vol. 20, no. 10, pp. 994-1003, 2014.

[28] G. Domes, E. Kumbier, M. Heinrichs, and S. C. Herpertz, "Oxytocin promotes facial emotion recognition and amygdala reactivity in adults with asperger syndrome," Neuropsychopharmacology, vol. 39, no. 3, pp. 698-706, 2014

[29] K. Lawrence, R. Campbell, and D. Skuse, "Age, gender, and puberty 


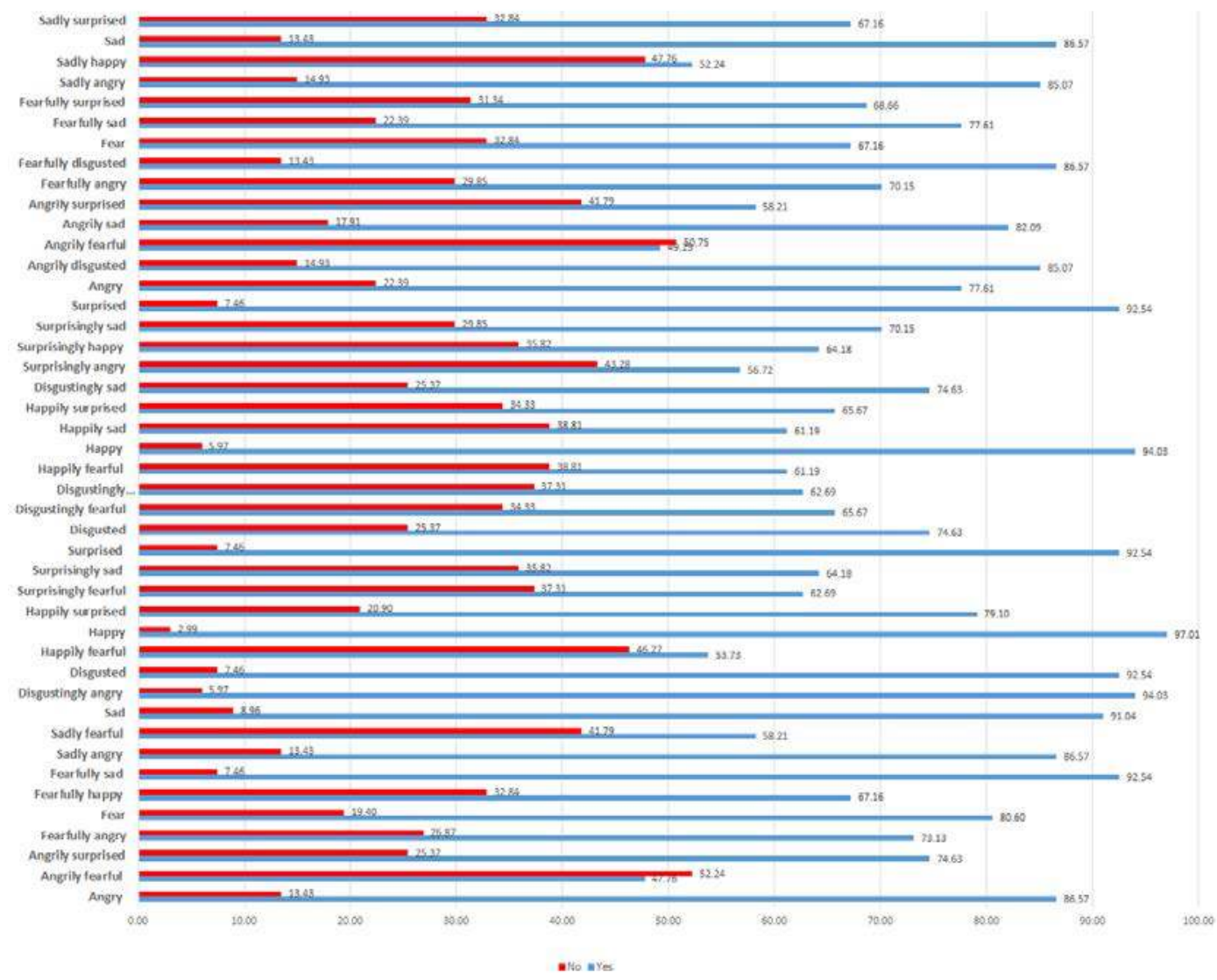

Fig. 7. The average result of the voting of 64 randomly chosen people on whether the facial emotion image is tagged correctly with the recognised emotion. The blue bar shows the percentage of the people who agreed with the recognised emotion and the red bar shows the percentage of the people who disagreed with the recognised emotion.

influence the development of facial emotion recognition," Frontiers in psychology, vol. 6, 2015.

[30] G. Hipp, N. J. Diederich, V. Pieria, and M. Vaillant, "Primary vision and facial emotion recognition in early parkinson's disease," Journal of the neurological sciences, vol. 338, no. 1, pp. 178-182, 2014

[31] G. M. M. Y. P. C. Valstar, Almaev, "Fera 2015 - second facial expression recognition and analysis challenge," Automatic Face and Gesture Recognition $(F G), 2015$ 11th IEEE International Conference and Workshops, vol. 6, 2015.

[32] M. Baltrusaitis, Robinson, " $3 \mathrm{~d}$ constrained local model for rigid and non-rigid facial tracking," Proceedings of the 2012 IEEE Conference on Computer Vision and Pattern Recognition (CVPR), 2012.

[33] Z. Zhang, "Feature-based facial expression recognition: Sensitivity analysis and experiments with a multilayer perceptron," International journal of pattern recognition and Artificial Intelligence, vol. 13, no. 06, pp. 893-911, 1999.

[34] S. Afzal, T. M. Sezgin, Y. Gao, and P. Robinson, "Perception of emotional expressions in different representations using facial feature points," in Affective Computing and Intelligent Interaction and Workshops, 2009. ACII 2009. 3rd International Conference on. IEEE, 2009, pp. 1-6.

[35] J. Viola, "Rapid object detection using a boosted cascade of simple features," Computer Vision and Pattern Recognition, 2001, vol. 1, 2001

[36] G. C. H. W.Zhang, Shan, "Local gabor binary pattern histogram sequence (lgbphs): a novel non-statistical model for face representation and recognition," Computer Vision, 2005. ICCV 2005, vol. 1, 2005.

[37] C.-W. Hsu, C.-C. Chang, and C.-J. Lin, "A practical guide to support vector classification," Department of Computer Science,
National Taiwan University, Tech. Rep., 2003. [Online]. Available: http://www.csie.ntu.edu.tw/ cjlin/papers.html

[38] N. Galvo, Fernandez-Martin, "Facial expression recognition in peripheral versus central vision: role of the eyes and the mouth," Psychological Research, vol. 78, 2014.

[39] A. Calder, A. Young, J. Keane, and M. Dean, "Configural information in facial expression perception," Journal of Experimental Psychology: Human Perception and Performance, vol. 26, no. 2, pp. 527-551, 2000.

[40] C. Blais, C. Roy, D. Fiset, M. Arguin, and F. Gosselin, "The eyes are not the window to basic emotions," Neuropsychologia, vol. 50, no. 12, pp. 2830-2838, 2012.

[41] F. A. G. Blais, Roy, "The eyes are not the window to basic emotions," Neuropsychologia, 2012.

[42] Z. Ambadar, J. F. Cohn, and L. I. Reed, "All smiles are not created equal: Morphology and timing of smiles perceived as amused, polite, and embarrassed/nervous," Journal of nonverbal behavior, vol. 33, no. 1, pp. 17-34, 2009.

[43] T. Kanade, J. F. Cohn, and Y. Tian, "Comprehensive database for facial expression analysis," in Automatic Face and Gesture Recognition, 2000. Proceedings. Fourth IEEE International Conference on. IEEE, 2000, pp. 46-53.

[44] M. N. Dailey, C. Joyce, M. J. Lyons, M. Kamachi, H. Ishi, J. Gyoba, and G. W. Cottrell, "Evidence and a computational explanation of cultural differences in facial expression recognition." Emotion, vol. 10, no. 6, p. $874,2010$.

[45] M. Lyons, S. Akamatsu, M. Kamachi, and J. Gyoba, "Coding facial expressions with gabor wavelets," in Automatic Face and Gesture Recognition, 1998. Proceedings. Third IEEE International Conference on. IEEE, 1998, pp. 200-205. 